\title{
La importancia de la educación física para el correcto desarrollo físico y cognitivo en la infancia
}

\section{The importance of physical education for proper physical and cognitive development in childhood}

DOI: $10.46932 / \mathrm{sfjdv2n2-091}$

Received in: March 1st, 2021

Accepted in: May 30th, 2021

\author{
Juani González Muñoz \\ Graduated as an elementaryschool teacher, \\ Institution: RG Formación \\ Calle Rosalía de Castro, 44, 30107 Murcia \\ Belén Cánovas Calderón \\ Graduated as an elementaryschool teacher, \\ Institution: RG Formación \\ Calle Rosalía de Castro, 44, 30107 Murcia \\ Rocío Muñoz Melgar \\ Graduated as an elementaryschool teacher, \\ Institution: RG Formación \\ Calle Rosalía de Castro, 44, 30107 Murcia \\ Jose María Rabal Alonso \\ Professor ISEN Centro Universitario \\ Institution: Universidad de Murcia \\ Calle Campus Universitario, 12, 30100 Murcia \\ E-mail: josemaria.rabal@um.es
}

\section{RESUMEN}

La práctica de las actividades físico deportivas ha alcanzado especial relevancia en los últimos años como factor determinante para conseguir un estilo de vida saludable y adquirir hábitos beneficiosos para la salud. Además de ello, el desarrollo y la promoción de las actividades físicas contribuye a un mejor bienestar emocional, así como a una mayor promoción de la salud. Adquirir hábitos saludables tales como mantener una buena alimentación, ser constantes con la higiene personal o postural, evitar el sedentarismo o el consumo de sustancias perjudiciales para la salud es una necesidad de primer orden que debe comenzar a inculcarse desde las edades más tempranas.

La asignatura de Educación Física y los profesionales especialistas en este ámbito educativo juegan un papel importante como agentes formadores que contribuirán a que los menores consigan todo lo anteriormente descrito. Para ello, es fundamental hacer un tratamiento global, transversal e integral de la asignatura, de manera que los alumnos consigan aprendizajes duraderos y significativos que poder aplicar en la vida cotidiana.

A lo largo del artículo se expondrá la experiencia y reflexión dos compañeros de profesión como alumnos de Educación Física, en la que se narra lo positivo y lo negativo de la vivencia. Este tipo de entrevistas ayudan a mejorar futuras sesiones o propuestas didácticas, evitando reproducir antiguos errores de los sistemas educativos pasados. Asimismo, se procederá a la definición de la Educación Física desde una perspectiva integral, enumerando sus finalidades y las actitudes que se pretenden desarrollar con la misma. 
Seguidamente se presentarán las diferentes técnicas o corrientes actuales que se imparten dentro de esta disciplina mediante una explicación y análisis de los puntos débiles que como docentes habrá que tener en cuenta. Cuerpo y motricidad son dos conceptos fundamentales cuando hablamos de esta área y es por ello que se tratarán profundamente a lo largo de este artículo. Estos elementos tan característicos y distintivos pueden conformar la identidad a nivel individual y colectivo mediante las sesiones de esta asignatura.

Por último, no se debe olvidar el tratamiento de la Educación Física como elemento fundamental para la formación integral de nuestros estudiantes, por ello hablaremos de los valores transversales y se harán propuestas de actividades, además de analizar algunos elementos del currículo al cual deben acogerse las actividades que se lleven a la práctica.

Palabras clave: salud, higiene, deporte, alimentación, obesidad

\begin{abstract}
The practice of physical sports activities has achieved special relevance in recent years as a determining factor in achieving a healthy lifestyle and acquiring habits beneficial to health. In addition, the development and promotion of physical activities contributes to a better emotional well-being, as well as to a greater promotion of health. Acquiring healthy habits such as maintaining a good diet, being consistent with personal or postural hygiene, avoiding a sedentary lifestyle or the consumption of substances harmful to health is a first-order need that should begin to be inculcated from the earliest ages. The subject of Physical Education and the professionals specializing in this educational field play an important role as training agents that will help children to achieve all of the above. For this, it is essential to make a global, transversal and integral treatment of the subject, so that students achieve lasting and significant learning that can be applied in everyday life.

Throughout the article, the experience and reflection of two colleagues as students of Physical Education will be presented, in which the positive and negative aspects of the experience will be narrated. This kind of interviews help to improve future sessions or didactic proposals, avoiding to reproduce old mistakes of past educational systems. Likewise, a definition of Physical Education will be made from an integral perspective, listing its purposes and the attitudes that are intended to be developed through it. Next, the different techniques or current currents that are taught within this discipline will be presented through an explanation and analysis of the weak points that, as teachers, we will have to take into account. Body and motor skills are two fundamental concepts when we talk about this area and that is why they will be deeply treated throughout this article. These characteristic and distinctive elements can shape the identity at an individual and collective level through the sessions of this subject.

Finally, we should not forget the treatment of Physical Education as a fundamental element for the integral formation of our students, that is why we will talk about the transversal values and proposals of activities will be made, besides analyzing some elements of the curriculum to which the activities to be put into practice must adhere.
\end{abstract}

Key words: health, hygiene, sport, nutrition, obesity.

\title{
1 VIVENCIA DE LA EDUCACIÓN FÍSICA Y COMENTARIO REFLEXIVO.
}

Comenzamos el artículo preguntando a una pareja de compañeros de profesión cómo fueron sus vivencias en su etapa estudiantil con respecto a la asignatura Educación Física (en adelante EF). Partimos con la siguiente pregunta ¿Cómo han sido tus vivencias en clase de EF?, como una primera toma de contacto y para familiarizarnos con el concepto de la asignatura y saber qué entendemos sobre ello y su mecánica, a partir de estas experiencias personales. 
“'Mi compañera me comenta que durante su etapa de Primaria la Educación Física es concebida como una hora de diversión en la que los compañeros interactúan y juegan constantemente al mismo tiempo que aprenden conceptos sobre el cuerpo humano o las indicaciones básicas de izquierda, derecha, arriba o abajo... en definitiva, su recuerdo es bastante bueno y positivo.

Sin embargo, durante la etapa de Educación Secundaria Obligatoria (E.S.O.), no pudo realizar Educación Física por problemas de salud. Todos los años a principio de curso llevaba un justificante médico con el cual quedaba exenta de realizar cualquier actividad física. Permanecía sentada con el profesor en la mayoría de ocasiones tomando notas o como ayudante del mismo para preparar las sesiones, circuitos, toma de notas etc. Lo que sí realizaba al igual que sus compañeros eran los exámenes teóricos, por lo que el profesor la evaluaba de manera exclusiva con esas calificaciones.

Afortunadamente, en la actualidad ha superado este problema, por tanto, actualmente puede realizar cualquier actividad deportiva con normalidad.

En cuanto a mi experiencia, la asignatura de Educación Física durante la etapa de Primaria la recuerdo con cariño. Se trataba de clases divertidas en las que primaba la construcción y el aprendizaje grupal. En líneas generales éramos una clase con buen comportamiento y eso hacía que los profesores nos premiaran algunas veces con salidas a los alrededores del colegio para hacer senderismo y estar en contacto con la naturaleza, ya que el centro estaba rodeado de huerta y terreno campestre. La mayoría de las veces realizábamos juegos en los que participábamos todos y en los que se evitaba la eliminación de compañeros durante el mismo.

En el instituto la asignatura deja de cobrar el mismo interés. Durante la E.S.O. la mayoría de las sesiones son dedicadas a deportes como el fútbol, baloncesto o bádminton, además de entrenar las pruebas de velocidad, resistencia, fuerza y flexibilidad, que posteriormente al final de trimestre serían evaluadas. Se trataba de clases monótonas y aburridas desde mi punto de vista.

Finalmente, considero que la excesiva deportivización de las sesiones de Educación Física o la valoración exclusiva de ciertas pruebas específicas para calificar a los alumnos, no son el camino para que estos disfruten y comprendan la importancia de la asignatura, ya que no solo debe buscar el entrenamiento o evaluación de ciertas habilidades o capacidades motrices, sino que debe ir más allá, buscando la educación integral y contribuyendo en todos los aspectos (afectivo, social, biológico, cognitivo etc.) del alumno'.

\section{INTRODUCCIÓN AL CONCEPTO DE EF.}




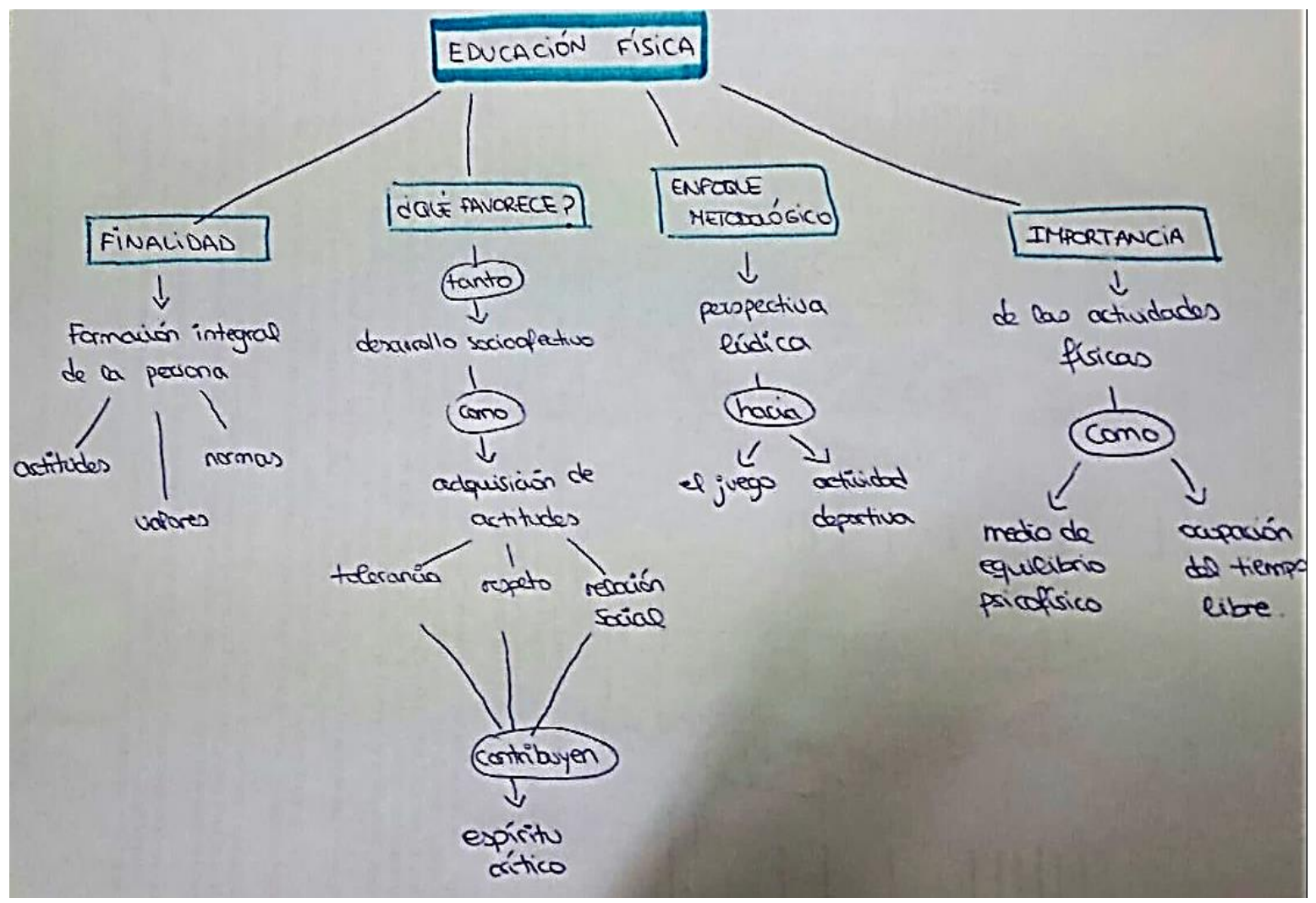

Como se ha mencionado en el punto anterior, la Educación Física debe contribuir no solo a las aptitudes físicas o condiciones de los alumnos, sino también a desarrollar en el alumno una formación integral, siendo este el principal objetivo de la asignatura. Para ello el docente debe ofrecer propuestas que favorezcan la formación de un espíritu crítico con actividades dirigidas hacia los intereses de los alumnos.

Durante la etapa de primaria la principal estrategia debe ser el juego, es decir, el profesor debe adoptar una perspectiva lúdica. La razón se debe a que en edades tan tempranas los alumnos buscan la diversión y a través del juego el alumno aprende valores como la tolerancia, el respeto hacia compañeros, forman y consolidan su carácter y, además, estimulan su poder creador gracias a su ingenio. Todas estas habilidades se obtienen de manera inconsciente a través de los juegos, por tanto, los alumnos aprenden sin apenas darse cuenta. En el aula también pueden ser capaces de aprenderlo, pero es cierto que fuera de ella los alumnos se sienten más libres, y esto facilita al docente de EF el desarrollo socio afectivo entre compañeros.

Cabe destacar, que los docentes de EF no son especialistas en salud en general, ni en dietética, nutrición, hábitos posturales, enfermedades u otras cuestiones en particular. Sin embargo, sí debemos ser conscientes de la importancia de su papel en la escuela como promotores de hábitos de vida saludables entre el alumnado, evitando el sedentarismo, la mala alimentación etc. En este sentido, aunque los contenidos de tipo conceptual son importantes, lo son aún más si cabe aquellos de carácter procedimental 
y actitudinal, y la educación física juega un papel muy importante en ello. Por esto, se pretende conseguir un equilibrio psicofísico en el alumno y una buena ocupación del tiempo libre en última instancia.

\section{TÉCNICA ACTIVA DE LAS CORRIENTES ACTUALES EN EF.}

A continuación, se lleva a cabo un pequeño análisis de cada una de las corrientes actuales en Educación Física, comentando qué aspectos trata cada una de ellas y señalando en la medida de lo posible, sus puntos débiles.

\subsection{HABILIDADES MOTRICES BÁSICAS}

Esta corriente americana clasifica las habilidades básicas en: movimientos que implican locomoción como los saltos o giros, movimientos que implican locomoción y manipulación como transportes o conducciones, movimientos que indican manipulaciones como lanzamientos o recepciones y, por último, las equilibraciones. Esta corriente contiene 'una importancia básica en el desarrollo motor de los niños y puede llegar a constituir uno de los ejes vertebradores de muchas de las actividades físicas en la escuela', (Lucea, 1999, p. 14). Sin embargo, esta corriente ha recibido numerosas críticas por su excesivo enfoque hacia lo deportivo. 


\subsection{DEPORTE}

Hay tres grandes orientaciones en esta corriente, deporte educativo, como medio para la integración de valores; deporte recreativo que piensa en la satisfacción de necesidades de movimiento del alumno desde un clima lúdico total y deporte institucional, que se observa en la integración del federacionismo deportivo. Este último ha sido muy criticado por tratar de conseguir bases deportivas especializadas por encima del objetivo educativo.

No obstante, sus clasificaciones son muy numerosas. Por ejemplo, se puede establecer la clasificación distinguiendo entre deportes individuales, deportes de oposición, deportes de colaboración y deportes de colaboración con oposición.

\subsection{EXPRESIÓN CORPORAL}

Atendiendo a la definición de Romero (2015), entendemos las actividades corporales de expresión como aquellas manifestaciones sociales y culturales, cuya finalidad es la expresión y comunicación a través del propio cuerpo y sus movimientos, así como el sentimiento como medio para guiar a los instrumentos básicos (cuerpo y movimiento)

Encontramos tres manifestaciones principales dentro de esta corriente: ritmo y danza, representación, dramatización y leguaje y bailes populares. El punto débil que presenta este concepto es la poca formación en este sentido que hayan podido recibir los docentes y profesionales de la educación.

\subsection{JUEGOS MOTORES}

Los juegos motores se justifican como contenido educativo por sí mismos, siempre que su orientación pedagógica sea educativa. Hay tres tipos principales: los juegos motores menores, formas jugadas o casi-juegos, los juegos recreativos y populares y los juegos deportivos.

Hay que tener en cuenta a la hora de llevar esta corriente al aula que la excesiva utilización del juego motor puede perder de vista el objetivo educativo. La EF va más allá del mero hecho de entretener y divertir, por tanto, el abuso de contenidos lúdicos sin otro fin que divertir, hace que la EF pierda el carácter educativo.

\subsection{ACTIVIDAD FÍSICA PARA LA SALUD}

Esta corriente es una de las más potenciadas de los últimos años, podemos observar en líneas generales tres enfoques dentro de esta corriente: preventiva, para disminuir malos hábitos y controlar los riesgos en el ejercicio y prevenir lesiones; compensatoria, aquella que rehabilita y adapta actividades que van dirigidas a las deficiencias orgánicas, musculares o de coordinación que puedan presentarse y como 
lucha contra el sedentarismo escolar, y condición física, que desarrolla y prepara las actividades físicas básicas, deportivas y cotidianas (resistencia, flexibilidad, fuerza, velocidad).

Esta corriente ha sido criticada en ocasiones por una especialización excesiva en los programas de intervención, ya que se pueden diferenciar bloques de contenidos de salud, cuando estas directrices deben seguirse en todas las intervenciones de EF.

\subsection{ACTIVIDAD EN LA NATURALEZA}

En los últimos años se ha convertido en una corriente de gran influencia en la EF. Esta corriente viene marcada por actividades al aire libre como acampadas o itinerarios, deportes en la naturaleza y juegos y actividades en la naturaleza.

Este tipo de actividades, demanda que los profesionales de la educación adapten las actividades al entorno en el que se lleven a cabo, así como una previa adaptación de los recursos a utilizar en cada una de ellas.

Para concluir con las corrientes de la EF, se considera que lo ideal es encontrar el equilibrio dentro y fuera de cada una. Es decir, se deben abordar todas en la etapa de Primaria, aprovechando sus puntos fuertes y disminuyendo los débiles. Además, estas no tienen por qué emplearse de manera aislada, sino que se pueden complementar las unas con las otras creando así sesiones de Educación Física verdaderamente enriquecedoras y de las que se pueda sacar el máximo provecho. De hecho, es muy difícil emplearlas por separado, ya que resultaría muy extraño emplear juegos motores sin una adecuada prevención física para la salud o realizar algún deporte sin llevar a cabo ciertas habilidades motrices, por ejemplo.

\section{CUERPO Y MOTRICIDAD.}

El cuerpo es un organismo biológico que se constituye por un conjunto de órganos que cumplen determinadas funciones, por lo tanto, la parte integrante del ser humano, mientras que la motricidad o movimiento es una de las manifestaciones del mismo, lo que se convierte en el objeto de estudio de la Educación Física, concretamente, las conductas motrices.

Podemos decir que el cuerpo tiene un dualismo (cuerpo anatómico o fisiológico y mente, alma o espíritu). Además, supone un encuentro entre lo individual y lo social, tiene mucho que ver con la naturaleza y la cultura, lo fisiológico (cuerpo) y lo simbólico (mente).

La influencia de ambos en la Educación Física es significante, pues el cuerpo no entiende de edad, sexo, cultura o bagaje, por ello es también que el estudio del cuerpo tiene gran influencia social, ya que 
adquirimos gestos, hábitos y formas de sensibilidad y de relación que la sociedad asigna a todas las personas.

Según Gómez (2009), la educación física es una disciplina pedagógica que debería ocuparse de crear las condiciones para el desarrollo de la corporeidad y la motricidad, para dar respuesta a las necesidades del sujeto que aprende, en un contexto determinado y en una sociedad cambiante, con conflictos de clase y culturas diferentes. Para ello se requiere una orientación de la educación física que parta del sujeto que necesita de los otros para desarrollar su potencial motriz y de aprendizaje.

A continuación, hablaremos de cuerpo social y subjetivo y su importancia en el área de Educación Física. En primer lugar, el cuerpo subjetivo rompe con la dualidad mente-cuerpo, y, como señala Scheler (1982) 'vuelve a considerarse cuerpo entero', por tanto, hablamos de un concepto unitario. Actualmente representa una introspección o análisis de lo vivido y las experiencias propias. Cuando interactuamos con nuestros iguales, el cuerpo adquiere una construcción social y cambia influenciado por la cultura. Es por ello que ocurre un movimiento, que tiene que ver con nuestra fisiología, biomecánica y anatomía. Además, de acuerdo a ello y según nuestras experiencias y forma de ser, mostramos diferentes esquemas personales y es por ello que damos lugar a una enorme dimensión comunicativa basada en la creatividad y el movimiento. Todo ello es posible siempre y cuando exista una estructura lógica relacional en cada uno.

\section{ANÁLISIS DE LAS CONTRIBUCIONES DE LA EF A LA EDUCACIÓN INTEGRAL DE LOS} ALUMNOS.

A continuación, se muestra el resultado de la búsqueda de un artículo sobre el trabajo en valores de Educación Física así como el resumen de los aspectos clave del mismo. El artículo encontrado se llama Propuesta de un programa para educar en valores a través de la actividad física y el deporte, y surge como fruto de una tesis doctoral. Destaca que, aunque la educación en valores se puede abordar desde muchas materias, la actividad física y el deporte son un contexto en el que se facilitan numerosas situaciones de relación entre compañeros y profesores, ofreciendo oportunidades únicas para desarrollar cualidades personales y sociales como la autoestima, la solidaridad, la cooperación etc.

Pero para llevar a cabo una verdadera educación en valores, es necesaria una metodología, objetivos, actividades y estrategias adecuadas propuestos por el docente, ya que las situaciones que se han comentado anteriormente también pueden desencadenar conflictos entre alumnos.

Las bases teóricas en las que se fundamenta el programa tienen como fuente principal el Modelo de Donald Hellison. Se estructura en tres fases:

La primera consiste en la confianza y la participación, donde se establecen unas bases mínimas que incluye el establecimiento de un ambiente positivo con interacciones afectivas y la introducción de 
objetivos (reglas, responsabilidades de los alumnos...) para iniciar la intervención. En esta fase damos a conocer a los alumnos el grado de implicación que se busca en ellos. Además, un segundo aspecto clave es la participación que queremos conseguir según el tipo de actividad que sea (deportes alternativos, tradicionales, juegos cooperativos, actividades al aire libre u otras).

Con la segunda fase damos paso a un trabajo específico en la transmisión de valores que queremos que se conviertan en referente. Aquí se incluyen el autocontrol de las conductas agresivas y violentas y para ello debemos organizar estratégicamente la composición de los equipos y grupos. Para ello debemos tener claro una serie de normas a establecer.

Por último, la tercera fase es una transferencia de lo aprendido a a otros ámbitos de la vida cotidiana, a su realidad más cercana y personal- para ello a lo largo de toda la intervención, intentaremos reflexionar y dialogar con los alumnos, haciéndoles entender que las estrategias planteadas tienen el objetivo de facilitar el bien común.

El artículo concluye destacando la necesidad de implantar programas de educación en valores, ya que los alumnos son el futuro y debemos implicarnos de lleno para mantener una sociedad pacífica sin arriesgar estas futuras generaciones, privándolas del conocer los beneficios que esto conlleva para todos y cada uno de ellos.

Una vez finalizado el análisis del artículo, procederemos al análisis esta vez de los valores y cómo se introducen en el currículo educativo actual. En primer lugar, los valores son cualidades o normas de conducta y actitudes, son los principios que adquieren las personas para el buen funcionamiento y bienestar de la sociedad.

El centro es colar es un buen escenario para la promoción de estos y es por eso que hoy en día es de capital importancia introducirlos en los bloques de contenidos curriculares. En todos los centros existe un Proyecto educativo que se regirá por lo dispuesto en el artículo 121 de la Ley Orgánica 2/2006, en el que entre otras cosas se hace referencia al tratamiento transversal de la educación en valores en todas las áreas y etapas.

La transversalidad curricular es, según Moreno (1999) "el conjunto de características que distinguen a un modelo curricular cuyos contenidos y propósitos de aprendizaje van más allá de los espacios disciplinares y temáticos tradicionales." Por tanto, se introducen de manera acorde con la etapa y área y produciéndose un encuentro entre estos y los contenidos específicos de cada asignatura. Muchos colegios además realizan multitud de actividades complementarias o extraescolares para contribuir a la consecución de valores como la igualdad de género, los hábitos de vida saludables, el respeto hacia el medio ambiente, la no violencia y respeto a los compañeros etc. 
Las sesiones de Educación Física nos ofrecen una gama amplia de oportunidades para trabajar los valores en Primaria. A continuación, se enumerarán algunos ejemplos:

Por ejemplo, la educación ambiental. En EF las relaciones con el entorno son muy ricas y podemos hacerlo ver a los alumnos reciclando objetos para que no se conviertan en basura y poder incluirlos como materiales de las actividades, promover la correcta ordenación y utilización de los vestuarios en lo referente al agua o electricidad o incluso haciendo senderismos o acampadas para la valoración del medio ambiente.

La educación para la paz también se puede conseguir con juegos en los que prime el diálogo, la cooperación y la búsqueda de una solución conjunta. Los juegos de colaboración-oposición también son una buena alternativa para promover el respeto y la tolerancia.

La educación sexual además de tratar los aspectos biológicos, puede ser introducida en EF para orientar y educar aspectos que van más allá, como son los aspectos afectivos, emocionales y sociales, entendiéndola como una actividad plena de comunicación entre las personas. Así como con la igualdad de género, haciéndoles ver a los alumnos que somos personas independientemente de nuestro sexo igualmente capacitadas para desempeñar distintos tipos de roles y actividades. Para ello la formación de equipos mixtos y los cambios de roles entre compañeros resultan positivos.

La educación para la salud en EF puede servirnos para orientar a los alumnos a llevar una vida sana, evitando los malos hábitos y creando aquellos que sean saludables en su vida escolar y cotidiana. Para ello, podemos indicar a los alumnos algunas pautas de alimentación, dieta y deporte que sean eficaces para ellos, así como indicarle las adecuadas posturas y normas a la hora de realizar ejercicios, juegos, estiramientos... de manera que no se hagan daño ni les perjudique.

Por último, aunque podíamos nombrar muchas más, la educación del consumidor es muy importante en EF. Muchos alumnos se ven sometidos (como la mayoría de los adultos) al impactante consumismo que existe hoy en día. Los niños, siempre quieren zapatillas o ropa deportiva de marcas caras o que estén promocionadas por sus ídolos, dejando de lado lo verdaderamente importante, hacer ejercicio y disfrutar de las sesiones y juegos preparados por el docente. Para ello podemos trabajar a través de juegos que promuevan la educación para la igualdad de oportunidades a partir de juegos en los que incluyamos equipos mixtos, sin distinción de razas, sexos, poder adquisitivo o creencias, en los que todos se perciban como iguales. Además, podemos utilizar materiales que históricamente hayan sido catalogados como de "niños" o de "niñas" y eliminar actitudes estereotipadas. En definitiva, y para cumplir con estos dos valores tan importantes, debemos hacer que los niños conozcan la importancia del "ser" y no del "tener". 
Asimismo, el deporte escolar puede propiciar situaciones de educación en valores. Además, la promoción de valores en el deporte no es una meta de la educación, sino una necesidad. A menudo, los niños juegan en deportes organizados por equipos, parejas, o individualmente. En algunos de los casos podemos observar la evidencia de valores de compañerismo y respeto a los adversarios, siendo prudentes muchos ellos de no perjudicar a los compañeros solo por conseguir una victoria. Pero si bien es cierto, los deportes institucionales que van más allá del centro escolar, desvirtúan un poco esos valores. Muchos niños son entrenados para ganar trofeos y ascender de categorías dejando de un lado el disfrute y entretenimiento.

Afortunadamente, el área de EF pretende contribuir en parte, en ver el deporte como una fuente de satisfacción personal y encuentro con los compañeros y no como una consecución obsesa de la victoria en la que no importa lo demás.

A continuación, se proponen cuatro actividades para trabajar la educación en valores en transversalidad en Educación Física.

Actividad 1: Los alumnos se colocarán por parejas en el centro del campo de espaldas al compañero. Una fila serán las conductas positivas y la otra, las negativas. El profesor dirá una acción y según sea positiva o negativa, el de la fila que corresponda tendrá que correr a pillar al otro antes de que llegue al final del campo. Las acciones negativas podrán ser burlarse, chillar, hacer trampas, enfadarse, insultar... y las positivas divertirse, participar, respetar, saber perder, cuidar el material etc. Esta actividad ayudará a los alumnos a distinguir entre las buenas y malas conductas.

Actividad 2: Los alumnos se dividirán en dos grupos, cada uno ensayará una actuación de mímica en la que ocurra algún problema, por ejemplo, un equipo puede representar un partido de fútbol en el cual hay un penalti, se marca el gol y el portero se enfada y da una patada al jugador. Una vez hayan terminado la representación, el otro equipo deberá mostrar la suya. Posteriormente pueden debatir los aspectos negativos y explicar cómo lo solucionarían ellos. Esta actividad puede servirles para adquirir conocimientos sobre cómo reaccionar en situaciones parecidas adoptando buenas conductas.

Actividad 3: En una bolsa se meterán unos papelitos que hagan alusión a diferentes valores. Los alumnos deberán agruparse en función del valor que les haya tocado, pero no podrán hablar en ningún momento para encontrar al resto de sus compañeros, tendrán que llegar a un entendimiento mediante gestos, sonidos etc.

Actividad 4: Los alumnos se sentarán en el suelo formando un circulo. El docente le dará una pelota a un alumno y otra a otro. Deberán pasarse la pelota en la misma dirección, pero sin 
que se lleguen a juntar las dos pelotas. A lo largo de la práctica se pueden ir introduciendo más pelotas. La colaboración y tolerancia con los compañeros juega un papel importante.

\section{EL CURÍCULUM EN EF.}

\subsection{CONTRIBUCIÓN DEL ÁREA DE EDUCACIÓN FÍSICA EN PRIMARIA.}

El área de Educación Física tiene como principal objetivo conseguir en el alumno una serie de aprendizajes que toman como referencia el ámbito motor y que son indispensables para su desarrollo pleno. Para su consecución es necesario el análisis crítico que afiance actitudes, valores referenciados al cuerpo, al movimiento y a la relación con el entorno. De este modo, el alumnado logrará controlar y dar sentido a las propias acciones motrices, comprender los aspectos perceptivos, emotivos y cognitivos relacionados con dichas acciones. Con ello, podrá gestionar los sentimientos vinculados a las mismas, además de integrar conocimientos y habilidades transversales, como el trabajo en equipo, el juego limpio y el respeto a las normas, entre otras.

\subsection{CONTRIBUCIÓN DEL ÁREA A LAS COMPETENCIAS BÁSICAS.}

La Educación Física está vinculada a la adquisición de competencias relacionadas con la salud través de acciones que ayuden a la adquisición de hábitos responsables de actividad física regular, y de la adopción de actitudes críticas ante prácticas sociales no saludables.

Además de los contenidos epistemológicos tratados en el área, la demanda social de crear ciudadanos competentes, determina que se debe formar a niños que sean capaces de comunicarse, de aplicar el pensamiento lógico-científico, de reflexionar, de emprender y crear, así como tener un bagaje y respeto cultural y un comportamiento cívico. El juego motor será imprescindible en esta etapa como situación de aprendizaje, acorde con las intenciones educativas, y como herramienta didáctica.

El área de Educación Física contribuye al desarrollo de las siete competencias delimitadas en el presente currículo, aunque la competencia social y cívica, la competencia para aprender a aprender y el sentido de la iniciativa y espíritu emprendedor, ocupan un lugar privilegiado entre los estándares de aprendizaje del área.

La aparición de estas competencias u otras en el desarrollo de las sesiones del área, va a depender del planteamiento docente, que ha de enfocar la práctica motriz para recoger información sobre estos comportamientos imprescindibles dentro de tareas que presenten problemas relevantes y reales, utilizando el contexto motor como medio para ello, sin menoscabo de la valoración de los aprendizajes motrices relacionados a continuación.

\subsection{BLOQUES DE CONTENIDOS.}


Teniendo en cuenta el Decreto $n^{\circ}$ 198/2014, de 5 de septiembre, por el que se establece el currículo de la Educación Primaria en la Comunidad Autónoma de la Región de Murcia, los contenidos referentes al área de Educación Física se han estructurado en cinco grandes bloques:

Bloque 1, El cuerpo. Imagen y percepción: enfocado al conocimiento de sí mismo, a la experimentación y dominio de las relaciones entre su cuerpo y el mundo exterior, poniéndolas en consonancia con la capacidad para organizar y planificar el movimiento y la disposición corporal requerida para ello.

Bloque 2, Habilidades motrices: diseñado para dotar y equipar al alumno de una serie de patrones motores, adquiridos de forma armónica y desde la perspectiva cualitativa.

Bloque 3, Actividades físicas artístico-expresivas: con el objeto de abordar aquellos elementos comunicativos, creativos y emocionales, relacionados directamente con la combinación existente entre los dos grandes ejes del área: el cuerpo y el movimiento.

Bloque 4, Actividad física y salud: este bloque aglutina un amplio abanico de conceptos, saberes prácticos y hábitos, destinados al fomento de una vida saludable desde una perspectiva integral.

Bloque 5, Juegos y actividades deportivas: destinados a dotar al alumno de una respuesta eficaz y diversificada, para la aplicación de los patrones motores adquiridos a entornos globales, así como mostrar una visión agradable y saludable de lo que supondría un ocio activo.

\subsection{ELABORACIÓN DE UNA SESIÓN DE EDUCACIÓN FÍSICA.}

La sesión será dividirá en tres partes:

La primera parte $(10 \mathrm{~min})$ irá dedicada a presentar la actividad a realizar. En ella se explicarán los objetivos de la sesión y las actitudes que queremos conseguir en los alumnos, así como incidir en los aspectos fundamentales relacionados con la actividad.

En la segunda parte (30-40 minutos) pasaremos a la práctica. Realizaremos una organización en feria en la que los alumnos, por parejas, pasarán por las diferentes estaciones. Aquellas podrán ser zancos, combas, mikados, juegos de mesa como el 3 en raya etc. 
Figura 2 .

Figura 3.
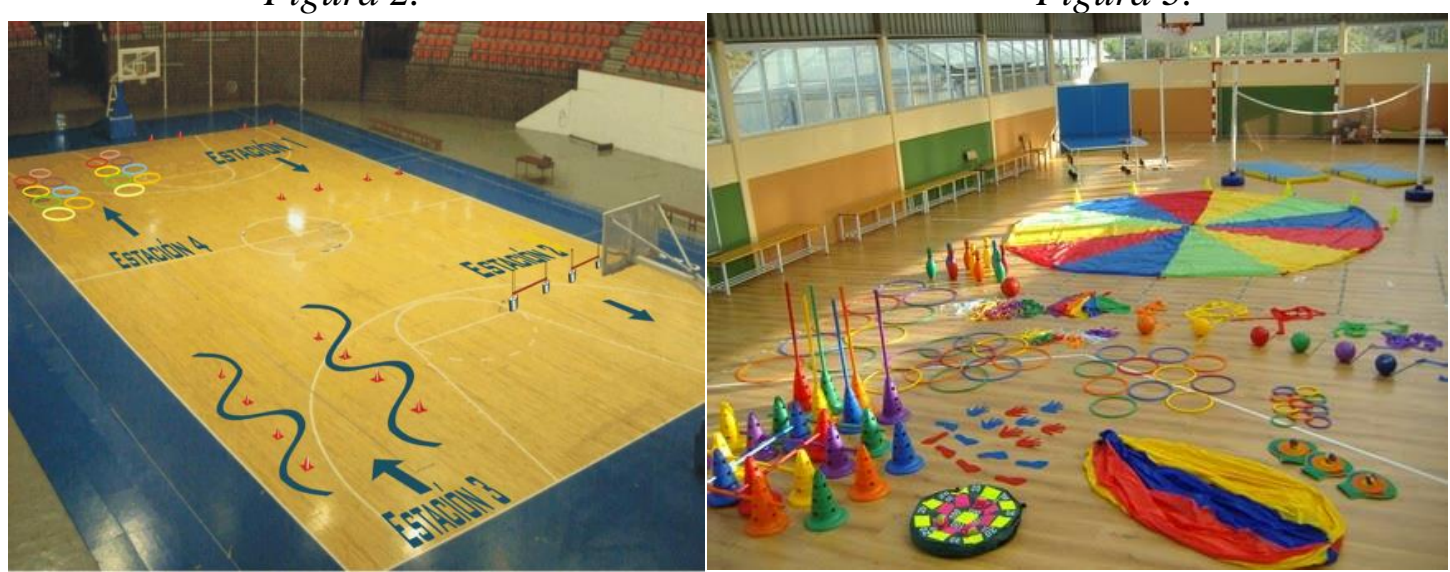

Por último, la tercera parte (10-20 minutos) será dedicada a una asamblea final en la que el profesor lanzará preguntas como: ¿Habéis pasado por todas las estaciones?, ¿Cuál habéis encontrado más divertida?, ¿Cuál os ha costado más?... así, conoceremos los resultados y pondremos solución a aquellos aspectos que puedan siempre mejorarse para posteriores sesiones. Esta asamblea también puede ser una herramienta para volver a la calma antes de la subida al aula, evitando que los alumnos lo hagan de forma atropellada o más intensa de la cuenta. Una vez hecho esto, los alumnos podrán ir a asearse.

\section{CONCLUSIÓN}

Para concluir, cabe destacar las numerosas capacidades y valores que se pueden trabajar y promover en una sesión de Educación Física. Esta asignatura ofrece a los alumnos muchas posibilidades de interacción y fomenta el desarrollo de habilidades que pueden resultar básicas en la vida social. De este modo, esta disciplina constituye un eslabón fundamental para la formación de individuos que se pretende que sean capaces de desenvolverse en la sociedad en la que vivimos.

Además de ello, se considera importante promover la adquisición del hábito físico-deportivo, el conocimiento del propio cuerpo y la expresión y comunicación que se puede realizar a través del mismo. Conseguir en los alumnos la práctica diaria deportiva por placer y no solo dentro del aula no es tarea fácil, pero utilizando metodologías innovadoras y lúdicas en las edades que comprenden la etapa de Educación Primaria se puede conseguir que los alumnos conciban el ejercicio y los hábitos saludables de manera favorable y olviden las connotaciones negativas que muchos de ellos poseen. Para conseguir esto último, es importante trabajar con el alumnado desde el respeto hacia nuestro cuerpo propio y el de los demás, siendo conscientes de las capacidades de cada compañero y fomentando la cooperación en la mayoría de actividades que se pongan en práctica. Una vez que los discentes comprendan el bienestar que nos aporta una adecuada actividad física, favoreceremos a la autonomía, estimularemos la creatividad y evitaremos 
el aislamiento social que se produce en muchas ocasiones por llevar una vida sedentaria y con hábitos perjudiciales.

Por último, es fundamental que los docentes de Educación Física además de tener las nociones básicas de la disciplina, tengan suficientes habilidades comunicativas, así como gran capacidad para motivar a su alumnado sin perder de vista el objetivo principal de las sesiones, manteniendo el orden y la buena conducta de los alumnos. Asimismo, en todas las áreas de la etapa, el buen docente debe mostrar sensibilidad ante los diferentes ritmos de aprendizaje, ya que no se debe olvidar que cada niño es único y por ende, las capacidades y ritmos de progresos también 


\section{BIBLIOGRAFÍA}

Alonso, J. M. R., \& Saorín, J. M. (2020). Compromiso motor en educación física en las aulas de educación primaria/Physical engagement and timing in physical education sessions in primary education. Brazilian Journal of Development, 6(4), 18714-18733.

Baena, A. (Coord.) (2009). Los temas transversales y su relación con el sistema educativo: Reflexiones y propuestas para docentes. AdaBook. Granada.

CARM (2014). Decreto 198/2014 de 5 de septiembre por el que se establece el currículo de la Educación Primaria de la Comunidad Autónoma de la Región de Murcia.

García J., Alonso, J.I., García, J.V., y López, P. (2011). Educación física en Educación Primaria I.

Gómez JR. Ministerio de Educación, Ciencia y Tecnología de la Nación. Dirección Nacional de Gestión Curricular y Formación Docente Áreas Curriculares - Educación Física (2008). La educación física y su contenido. Universidad de Flores, Argentina. Disponible en: http://www.me.gov.ar/curriform/publica/educ_fisica_gomez.pdf

Jiménez Martín, P. J. (2004). Propuesta de un programa para educar en valores a través de la actividad física $y$ el deporte. Recuperado de: https://www.raco.cat/index.php/ApuntsEFD/article/view/301453/391031

Lucea, J. D. (1999). La enseñanza y aprendizaje de las habilidades y destrezas motrices básicas (Vol. 133). Inde.

Martín, M. R. R. (2015). Expresión corporal en educación física (Vol. 245). Prensas de la Universidad de Zaragoza.

Scheler, M. (1982): El puesto del hombre en el cosmos. La idea de paz perpetua y el pacismo. Barcelona: Alba Editorial. 